\title{
Two-Step Enantio-selective Optical Switch
}

\author{
Petr Král ${ }^{1}$, Ioannis Thanopulos ${ }^{1}$, Moshe Shapiro ${ }^{1}$ and Doron Cohen ${ }^{2}$ \\ ${ }^{1}$ Department of Chemical Physics, Weizmann Institute of Science, Rehovot, Israel and \\ ${ }^{2}$ Department of Physics, Ben-Gurion University, Beer-Sheva, Israel
}

(Dated: November 3, 2018)

\begin{abstract}
We present an optical "enantio-selective switch", that, in two steps, turns a ("racemic") mixture of left-handed and right-handed chiral molecules into the enantiomerically pure state of interest. The optical switch is composed of an "enantio-discriminator" and an "enantio-converter" acting in tandem. The method is robust, insensitive to decay processes, and does not require molecular preorientation. We demonstrate the method on the purification of a racemate of (transiently chiral) $\mathrm{D}_{2} \mathrm{~S}_{2}$ molecules, performed on the nanosecond timescale.
\end{abstract}

PACS numbers: $33.15 . \mathrm{Bh}, 33.80 .-\mathrm{b} 42.50 . \mathrm{Hz} 42.62 .-\mathrm{b}$

Asymmetric synthesis [1] and chiral purification [2, 3, ㄴ.t of a ("racemic") mixture of enantiomers (chiral molecules and their mirror images) are among the most important and difficult tasks in chemistry. The possibility of achieving purification solely by optical means has been also theorized [5, 6, 7, 8]. In particular, this goal could be realized via a "laser distillation" scheme [8], in which a repetitive use of three light pulses [9] gradually purifies the system.

Recently, we suggested a method for achieving chiral separation, termed "Cyclic Population Transfer" (CPT) [10]. The approach is akin to the Adiabatic Passage (AP) [11, 12, 13], used to completely transfer population between quantum states, that are usually optically coupled as, $|1\rangle \leftrightarrow|2\rangle \leftrightarrow|3\rangle$. In chiral molecules, lacking an inversion center and thus having eigenstates with ill defined parity, it is possible to close the "cycle" by introducing a third field which couples the states $|1\rangle \leftrightarrow|3\rangle$ directly. The interference of one and two-photon transitions along the two paths renders the evolution, in this CPT scheme, dependent on the total phase $\varphi$ of the three (material+optical) coupling terms [10]. Since the transition dipoles of the two enantiomers differ in sign, the evolution in the two under the action of the three fields is different, and the enantiomers can be separated. In particular, if they initially occupy state $|1\rangle$, one enantiomer can be excited to state $|2\rangle$, while the other is transferred to state $|3\rangle$, or vice versa, depending on the phase $\varphi$.

The great advantage of the CPT scheme is that the separation process can be completed in just one step. We can do it using optical transitions, taking place on the ground electronic surface, thereby avoiding disruptive competing processes, such as dissociation and internal conversion 8]. However, CPT is not particularly robust with respect to variation of the laser pulses and it does not convert one enantiomer into another.

In this work, we demonstrate that optical chiral purification can be accomplished in just two (separation and conversion) steps, in a scheme which overcomes the above drawbacks. In the first one, called "enantiodiscriminator", we excite one enantiomer while leaving

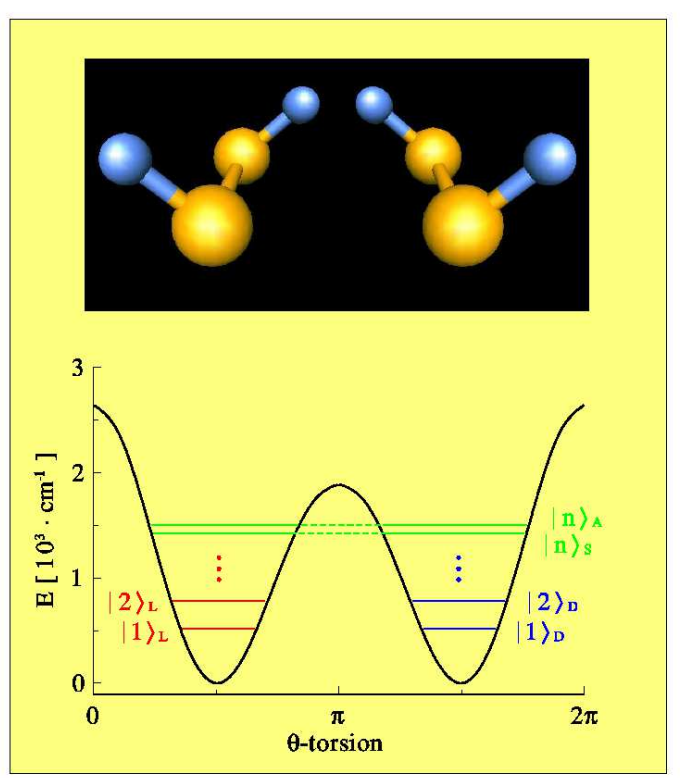

FIG. 1: The left and right $\mathrm{D}_{2} \mathrm{~S}_{2}$ enantiomers, which undergo stereomutation about the $S-S$ bond. Below is shown the double well potential energy for their torsional motion on the ground electronic state. In the considered $100 \mathrm{~ns}$ timescale, the lower energy (red and blue) ro-vibronic states, schematically shown here, have a definite (left and right) symmetry, while at higher energies they become split into symmetric and antisymmetric (green) states.

the other in its initial state. In the second one, called "enantio-converter", the enantiomer excited in the previous step is converted to its mirror-image form. Thus, in just two steps, the racemic mixture of chiral molecules could be converted into the enantiomer of choice.

We present the enantiomer switch on the (transiently chiral) $\mathrm{D}_{2} \mathrm{~S}_{2}$ molecule, shown schematically in Fig. 1 . The molecule has 6 vibrational degrees of freedom, with the large-amplitude torsional motion of the $\mathrm{D}$ (blue) atoms about the $\mathrm{S}-\mathrm{S}$ (orange) bond. A one-dimensional cut of the ground electronic potential energy surface along the enantio-mutative path is shown in Fig. 1 . 
From our ab-initio calculation, we have determined that the enantio-mutation of the molecules can be realized through a cis barrier $(\theta=0), 2700 \mathrm{~cm}^{-1}$ in height, or the trans barrier $(\theta=\pi), 1900 \mathrm{~cm}^{-1}$ in height. The calculated tunneling splitting of the lowest torsional states gives enantiomeric lifetimes of several msec, in accordance with previous reports on mode selective stereomutation [14]. Thus, although $\mathrm{D}_{2} \mathrm{~S}_{2}$ is not a chiral molecule in the conventional sense, molecular configurations described by superpositions of the lowest torsional states, localized in one minimum of the double well potential, stay chiral for sufficiently long times to be detected.

The two-step process makes use of five pairs of $D_{2} S_{2}$ ro-vibrational eigenstates. The vibrational states correspond to the combined torsional and $\mathrm{S}-\mathrm{D}$ asymmetric stretching modes. The rotational states correspond to a rigid rotor. Within the pairs used [15], each eigenstate has an $S / A$ (symmetric/antisymmetric) label denoting its symmetry with respect to inversion, and the chiral states with $L / D$ labels are composed as, $|k\rangle_{L, D}=$ $\frac{1}{\sqrt{2}}\left(|k\rangle_{S} \pm|k\rangle_{A}\right), \quad k=1, \ldots, 4$. The $|k\rangle_{L} \leftrightarrow|k\rangle_{D}$ interconversion period is $\tau_{s} \approx 33,3.3,0.165 \mathrm{~ms}$ for $k=1,2,3$, respectively, and $\tau_{s} \approx 0.05 \mu \mathrm{s}$ for $k=4$. The higher lying $|5\rangle_{S}$ and $|5\rangle_{A}$ states are separated by $\Delta E_{S, A}^{5}=0.38 \mathrm{~cm}^{-1}$, for which $\tau_{s} \approx 0.1 \mathrm{~ns}$, and can be thus separately addressed by $n s$ pulses, that are strong enough, to ensure adiabaticity. The ns pulses are also long enough to address individual lower lying ro-vibronic levels, but, since $\tau \ll \tau_{s}$, they cannot separately address the narrowly split $S$ and $A$ states, so the symmetrybroken combination states $|k\rangle_{L, D}(k=1-4)$ become physically meaningful.

We now describe in details the dynamics of the switch. Denoting the energy of level $|i\rangle$ by $\omega_{i}(\hbar=1$ in atomic units), we choose the external electric field to be a sum of components, each being in resonance with one of the $|i\rangle \leftrightarrow|j\rangle$ transition frequencies of interest, $\mathbf{E}(t)=$ $\sum_{i \neq j} \mathcal{R}_{e}\left[\hat{\epsilon} \mathcal{E}_{i, j}(t) e^{-i \omega_{i, j} t}\right]$, where $\omega_{i, j}=\omega_{i}-\omega_{j}$, and $\hat{\epsilon}$ is the polarization direction. The Hamiltonian of the system in the rotating wave approximation is,

$$
\mathrm{H}=\sum_{i=1}^{N} \omega_{i}|i\rangle\langle i|+\sum_{i>j=1}^{N}\left(\Omega_{i, j}(t) e^{-i \omega_{i, j} t}|i\rangle\langle j|+\text { H.c. }\right) \text {. }
$$

It depends on the Rabi frequencies, $\Omega_{i, j}(t)=\mu_{i, j} \mathcal{E}_{i, j}(t)$, where $\mu_{i, j}$ are the transition-dipole matrix elements. Expanding the system wave function in the material states $|i\rangle$ as, $|\psi(t)\rangle=\sum_{i=1}^{N} c_{i}(t) e^{-i \omega_{i} t}|i\rangle$, the (column) vector of the slow varying coefficients $\mathbf{c}=\left(c_{1}, c_{2}, \ldots, c_{N}\right)^{\mathrm{T}}$, with $\mathrm{T}$ designating the matrix transpose, is the solution of the matrix-Schrödinger equation $\dot{\mathbf{c}}(t)=-i \mathbf{H}(t) \cdot \mathbf{c}(t)$, where $\mathbf{H}(t)$ is an effective Hamiltonian matrix, given explicitly below for the two processes.

The scheme of the three-level "enantio-discriminator" is shown in the upper panel of Fig. 2. Assuming that the system is at low temperature, so that we can practically start with a mixture of chiral $\mathrm{D}_{2} \mathrm{~S}_{2}$ molecules in the ground $|1\rangle_{L}$ and $|1\rangle_{D}$ states, the task of the discriminator is to selectively transfer one enantiomer to the $|3\rangle$ state and to keep the other in the $|1\rangle$ state. Due to the degeneracy of the $|i\rangle_{L}$ and the $|i\rangle_{D}$ levels $(i=1,2,3)$, the field $\mathbf{E}(t)$ simultaneously excites the resonant $|i\rangle_{L, D} \leftrightarrow|j\rangle_{L, D}, i \neq j=1,2,3$ transitions of both enantiomers 9 .

In the first enantio-discriminator step, the effective Hamiltonian matrix is,

$$
\mathbf{H}(t)=\left[\begin{array}{ccc}
0 & \Omega_{1,2}^{*}(t) & \Omega_{1,3}^{*}(t) \\
\Omega_{1,2}(t) & 0 & \Omega_{2,3}^{*}(t) \\
\Omega_{1,3}(t) & \Omega_{2,3}(t) & 0
\end{array}\right] .
$$

The phases of the Rabi frequencies $\Omega_{i, j}(t)$ are given as in the CPT scheme by $\phi_{i, j}=\phi_{i, j}^{\mu}+\phi_{i, j}^{E}$, where $\phi_{i, j}^{\mu}$ are the phases of the dipole matrix elements $\mu_{i, j}$, and $\phi_{i, j}^{E}$ are the phases of the electric field components $\mathcal{E}_{i, j}$. The evolution of the system is determined [10] by the total phase $\varphi \equiv \phi_{1,2}+\phi_{2,3}+\phi_{3,1}$. This is most noticeable at the time $t=\tau$, for which the three Rabi frequencies are equal in magnitude, $\left|\Omega_{1,2}\right|=\left|\Omega_{1,3}\right|=\left|\Omega_{2,3}\right|=\Omega$. Denoting the eigenvalues of the Hamiltonian of Eq. (2) as $E_{-}$, $E_{0}$ and $E_{+}$, it is easy to show that they exhibit exact degeneracies (crossings) at $t=\tau$, with $E_{+}=2 \Omega$ and $E_{-}=E_{0}=2 \Omega \cos (2 \pi / 3)$, for $\varphi=0$, and $E_{-}=-2 \Omega$ and $E_{+}=E_{0}=-2 \Omega \cos (2 \pi / 3)$, for $\varphi=\pi$. Depending on the polarizations of the fields, one or all three Rabi frequencies $\Omega_{i, j}$ of the two enantiomers differ by a sign [10], making $\varphi$ differ by $\pi$ for the two. Therefore, the above two degeneracies (crossings) occur at different enantiomers, leading subsequently to their totally different dynamics, depicted in the lower panel of Fig. 2.

The overall enantio-discriminator works as follows: We start with a "dump" pulse $\mathcal{E}_{2,3}(t)$ that couples the $|2\rangle$ and $|3\rangle$ states and has the Rabi frequency $\Omega_{2,3}(t)=$ $\Omega^{\max } f(t)$, where $\Omega^{\max }=1 \mathrm{~ns}^{-1}$ and $f(t)=\exp \left[-t^{2} / \tau^{2}\right]$. At this stage of the process all the population resides in the $\left|E_{0}\right\rangle$ (adiabatic) eigenstate. In the second stage we $s i$ multaneously add two "pump" pulses of the Rabi frequencies $\Omega_{1,2}(t)=\Omega_{1,3}(t)=\Omega^{\max } f(t-2 \tau)$, that couple the $|1\rangle \leftrightarrow|2\rangle$ and the $|1\rangle \leftrightarrow|3\rangle$ states. We choose the phases of the optical fields such that $\varphi=0$ for one enantiomer and, inevitably, $\varphi=\pi$ for the other. Therefore, the population, which has been following in both enantiomers the initial adiabatic level $\left|E_{0}\right\rangle$, goes at $t=\tau$ smoothly through the crossing region and diabatically transfers to either the $\left|E_{-}\right\rangle$or the $\left|E_{+}\right\rangle$states, depending on whether $\varphi=0$ or $\varphi=\pi$, i.e., on the identity of the enantiomer.

After the crossing is complete, at $t>\tau$, the process becomes adiabatic again, with the enantiomer population residing fully in either $\left|E_{-}\right\rangle$or $\left|E_{+}\right\rangle$. At this stage we slowly switch off the $\mathcal{E}_{1,2}(t)$ pulse while making sure that the $\mathcal{E}_{1,3}(t)$ field remains on. This is done by choosing 

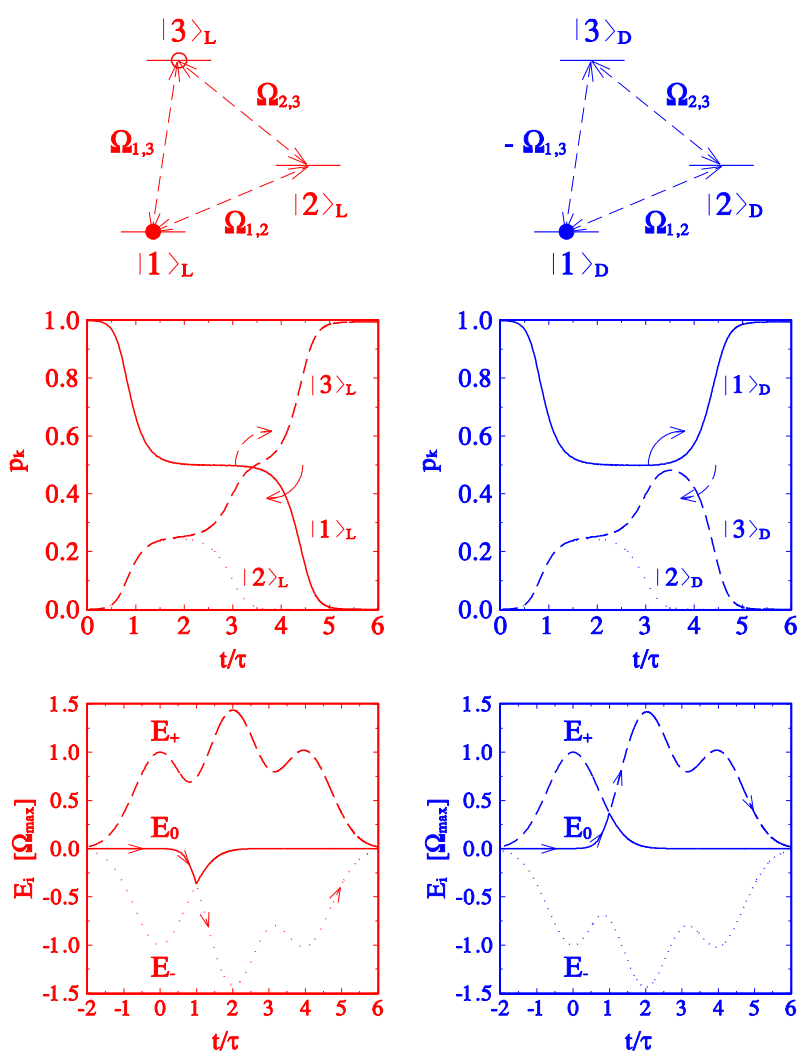

FIG. 2: (Upper panel) A schematic plot of the enantiodiscriminator. The three levels of each enantiomer are resonantly coupled by three fields. (Middle panel) The time evolution of the population of the three levels. Both enantiomers start in the $|1\rangle$ state. At the end of the process the $L$ enantiomer is transferred to the $|3\rangle_{L}$ state, while the $D$ enantiomer remains in the initial $|1\rangle_{D}$ state. (Lower panel) The time-dependence of the eigenvalues of the Hamiltonian of Eq. (2). The population initially follows the $\left|E_{0}\right\rangle$ dark state. At $t \approx \tau$ the population crosses over diabatically to $\left|E_{-}\right\rangle$for one enantiomer and to $\left|E_{+}\right\rangle$for the other.

$\Omega_{1,3}(t)=\Omega^{\max }\left(f(t-2 \tau)+f(t-4 \tau) \exp \left\{-i t \Omega^{\max } f(t-\right.\right.$ $6 \tau)\})$. As a result, the zero adiabatic eigenstate $\left|E_{0}\right\rangle$ correlates adiabatically with state $|2\rangle$, which thus becomes empty after this process, while the occupied $\left|E_{+}\right\rangle$and $\left|E_{-}\right\rangle$states correlate to, $\left|E_{ \pm}\right\rangle \rightarrow(|1\rangle \pm|3\rangle) / \sqrt{2}$.

The chirp, $\exp \left\{-i t \Omega^{\max } f(t-6 \tau)\right\}$, in the second term of $\Omega_{1,3}(t)$ causes a $\pi / 2$ rotation in the $\{|1\rangle,|3\rangle\}$ subspace at $t \approx 5 \tau$. As a result, state $\left|E_{+}\right\rangle$goes over to state $|3\rangle$ and state $\left|E_{-}\right\rangle$goes over to state $|1\rangle$, or vice versa, depending on $\varphi$. The net result of the adiabatic passage and the rotation is that one enantiomer returns to its initial $|1\rangle$ state and the other switches over to the $|3\rangle$ state. As shown in the middle panel of Fig. 2, the enantio-discriminator is very robust, in contrast to the CPT scheme [10], with all the population transfer processes occurring in a smooth fashion.

Assuming that the $L$ enantiomer has been excited to the $|3\rangle_{L}$ state, we now proceed to convert it into a $D$ enantiomer in the $|4\rangle_{D}$ state, by going through a linear superposition of $|5\rangle_{S}$ and $|5\rangle_{A}$ states, while leaving intact the $D$ enantiomer in the state $|1\rangle_{D}$. This "enantioconverter" process, based on a new multi-path transfer technique 16], thus schematically follows the pathway $|3\rangle_{L} \rightarrow \alpha e^{-i \omega_{5 S} t}|5\rangle_{S}+\beta e^{-i \omega_{5 A} t}|5\rangle_{A} \rightarrow|4\rangle_{D}$, shown in Fig. 3. The transfer is realized by simultaneously introducing two "dump" pulses $\mathcal{E}_{4,5 S}(t)$ and $\mathcal{E}_{4,5 A}(t)$ (of duration $\left.\tau \gg\left(\omega_{5 S}-\omega_{5 A}\right)^{-1}\right)$, which resonantly couple each of the $|5\rangle_{S}$ and $|5\rangle_{A}$ states to the $|4\rangle_{L}$ and $|4\rangle_{D}$ state. After a delay of $2 \tau$, we introduce two ("pump") pulses $\mathcal{E}_{3,5 S}(t)$ and $\mathcal{E}_{3,5 A}(t)$, which resonantly couple each of the $|5\rangle_{S}$ and $|5\rangle_{A}$ states to the $|3\rangle_{L}$ and $|3\rangle_{D}$ state. In this process only the $|3\rangle_{D}$ and $|4\rangle_{L}$ states are populated, while the $|3\rangle_{L}$ and $|4\rangle_{D}$ states, degenerate with them, respectively, stay empty. This is because the empty pair of states is coupled to the $|5\rangle_{S}$ and $|5\rangle_{A}$ states by vectors of Rabi frequencies $\Omega_{i}$, that are orthogonal [16] to analogous vectors of the populated pair of states, respectively. The symmetry conversion of the excited enantiomer is achieved by choosing $\Omega_{4,5 S}(t)$ to have the same sign as $\Omega_{3,5 S}(t)$ and $\Omega_{4,5 A}(t)$ to have an opposite sign to $\Omega_{3,5 A}(t)$.

The enantio-converter is described by the Hamiltonian,

$$
\mathbf{H}=\left[\begin{array}{cccccc}
0 & 0 & -\Omega_{3,5 S} & \Omega_{3,5 A} & 0 & 0 \\
0 & 0 & \Omega_{3,5 S} & \Omega_{3,5 A} & 0 & 0 \\
-\Omega_{3,5 S}^{*} & \Omega_{3,5 S}^{*} & 0 & 0 & -\Omega_{4,5 S}^{*} & \Omega_{4,5 S}^{*} \\
\Omega_{3,5 A}^{*} & \Omega_{3,5 A}^{*} & 0 & 0 & \Omega_{4,5 A}^{*} & \Omega_{4,5 A}^{*} \\
0 & 0 & -\Omega_{4,5 S} & \Omega_{4,5 A} & 0 & 0 \\
0 & 0 & \Omega_{4,5 S} & \Omega_{4,5 A} & 0 & 0
\end{array}\right]
$$

with the time-dependent wavefunction given by the vector $\mathbf{c}(t)=\left(c_{3 L}, c_{3 R}, c_{5 S}, c_{5 A}, c_{4 L}, c_{4 D}\right)$ of expansion coefficients in the $|i\rangle$ states. The Hamiltonian matrix (3) has four non-zero eigenvalues and two null eigenvalues, $\lambda_{1,2}=0$, that correspond to two dark states with the coefficients $\mathbf{c}_{1}(t)=\left(-d_{+},-d_{-}, 0,0,2 \Omega_{3,5 S}, 0\right), \mathbf{c}_{2}(t)=$ $\left(-d_{-},-d_{+}, 0,0,0,2 \Omega_{3,5 S}\right)$, where $d_{ \pm} \equiv \Omega_{4,5 S} \pm r \Omega_{4,5 A}$, with $r \equiv \Omega_{3,5 S} / \Omega_{3,5 A}$. These expressions show that the system can follow two possible paths, where only one of them is flipping the symmetry of the initial state. Assuming, for simplicity, that $r=1$ and $r^{\prime}=\Omega_{4,5 S} / \Omega_{4,5 A}=1$, we find out that at beginning of the process only the dark state $\mathbf{c}_{1}\left(t_{i n i}\right)$ correlates with the initial state $|3\rangle_{L}$, i.e. the vector $\mathbf{c}\left(t_{i n i}\right)=(1,0,0,0,0,0)$. At the end of the processes, this dark state correlates with the vector $\mathbf{c}_{1}\left(t_{\text {end }}\right)=(0,0,0,0,1,0)$ for the $|4\rangle_{L}$ state, so the symmetry is preserved. On the other hand, if we flip the phase of just one dump or one pump field component $\left(r=-1\right.$ or $\left.r^{\prime}=-1\right)$ the system follows the dark state $\mathbf{c}_{2}$, which correlates at the end with the state $\mathbf{c}_{2}\left(t_{\text {end }}\right)=(0,0,0,0,0,1)$. The final population thus occupies the $|4\rangle_{D}$ state, with the opposite symmetry. This brings the whole population to a single enantiomer form. 

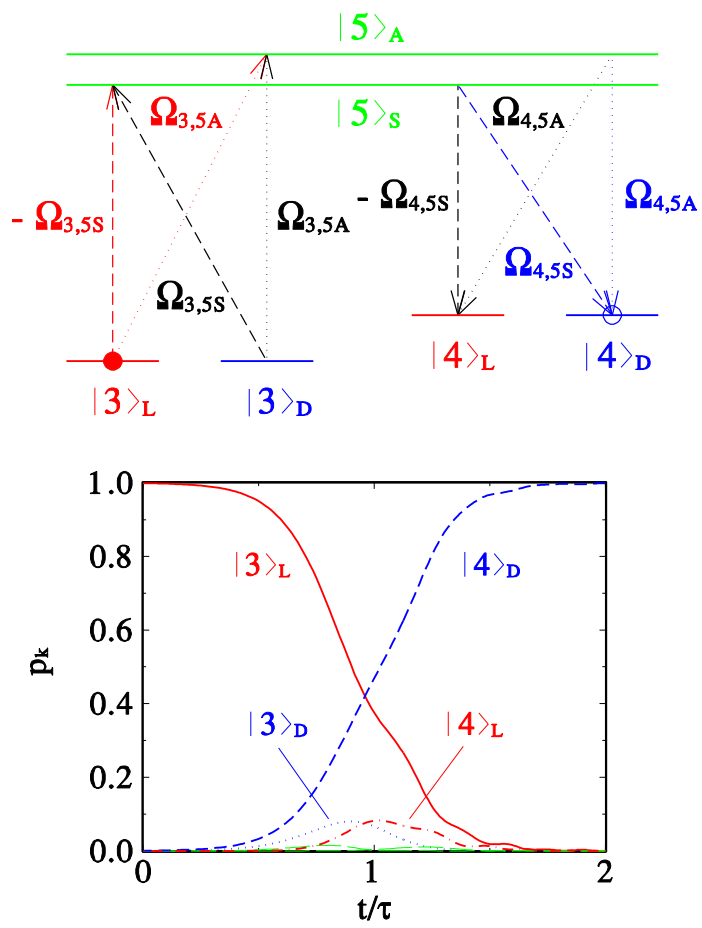

FIG. 3: (Upper plot) Scheme of the enantio-converter. The population passes from the $|3\rangle_{L}$ state to the $|4\rangle_{D}$ state, while going through the superposition of $|5\rangle_{S, A}$ states. (Lower plot) The time-dependent populations $p_{i}$ on the levels.

In Fig. 3, we show evolution of the calculated populations $p_{i}=\left|c_{i}\right|^{2}$. The process starts in the $|3\rangle_{L}$ state and ends in the $|4\rangle_{D}$ state. The Rabi frequencies are $\Omega_{3,5 S}(t)=\Omega^{\max } f(t-2 \tau), \Omega_{3,5 A}(t)=0.5 \Omega^{\max } f(t-2 \tau)$, $\Omega_{4,5 S}(t)=0.4 \Omega^{\max } f(t), \Omega_{4,5 A}(t)=-\Omega^{\max } f(t)$, with $\Omega^{\max }=30 \mathrm{~ns}^{-1}$ and $f(t)$ as in the discriminator. Notice, that we have rather different $r=2, r^{\prime}=-0.4$, which shows that this system is robust, i.e. it does not require that $|r|=1$ and $\left|r^{\prime}\right|=1$ to follow this complete-transfer path, but these ratios need to have the right sign.

Because the higher excited $|5\rangle_{S, A}$ states never get populated in the conversion, the switch is immune to $T_{2}$-like dephasing processes, which destroy the relative phase between the components of excited superposition states. For the same reason, it is insensitive to dissociation and/or internal conversion, even if higher electronic surfaces, having well separated $|5\rangle_{S, A}$ states, are used in truly chiral molecules. These states should have reasonably strong dipolar coupling with the $|3-4\rangle_{L, D}$ states, from the ground electronic surface, so that the light intensities are not too high for parasitic processes to take place. Then the only requirement is for the process to be over before a $T_{1}$ type collisional relaxation of the states takes place. Recently, we have successfully applied this approach on the permanently chiral molecule, that of 1,3dimethylallene.

In experiments, we can tune the laser parameters to match the molecular parameters, until the conversion process becomes effective. We can, for example, continuously check the product by sensitively testing its circular birefringence 17]. The method can be applied even if the conversion is not perfect from various reasons, like when several molecular states are initially populated at higher temperatures. Then the purification can be completed by repeating the described process several times with the same set of pulses. We just always let the system relax, so that the process starts with reasonable populations on the same initial states.

We believe that the new methodology presented here can largely influence the science and technology of chiral molecules purification, and lead to applications in organic chemistry, biochemistry and drug industry.

We acknowledge many discussions with P. Brumer and E. Frishman. This project was supported by the Minerva Foundation, GIF, the EU IHP program HPRN-CT-199900129, the Office of Naval Research, USA, and the Swiss Friends of the Weizmann Institute.

[1] W. S. Knowles (Nobel lecture 2001), Angew. Chem. Int. Ed. Eng. 41, 1997 (2002).

[2] K. Bodenhöfer, A. Hierlemann, J. Seemann, G. Gauglitz, B. Koppenhoefer and W. Göpel, Nature 387, 577 (1997).

[3] R. McKendry, M.-E. Theoclitou, T. Rayment and C. Abell, Nature 391, 566 (1998).

[4] H. Zepik et al., Science 295, 1266 (2002).

[5] M. Shapiro and P. Brumer, J. Chem. Phys. 95, 8658 (1991).

[6] A. Salam and W. J. Meath, Chem. Phys. 228, 115 (1998).

[7] Y. Fujimura, L. Gonzalez, K. Hoki, J. Manz and Y. Ohtsuki, Chem. Phys. Lett. 306, 1 (1999).

[8] M. Shapiro, E. Frishman and P. Brumer, Phys. Rev. Lett. 84, 1669 (2000).

[9] The linearly polarized electric fields should have mutually orthogonal polarizations. We thus also avoid deleterious averaging of the angular momentum directions (M. Shapiro, E. Frishman and P. Brumer, submitted).

[10] P. Král and M. Shapiro, Phys. Rev. Lett. 87, 183002 (2001).

[11] D. Grischkowsky and M. M. T. Loy, Phys. Rev. A 12, 1117 (1975). ibid, 2514 (1975).

[12] J. Oreg, F. T. Hioe, and J. H. Eberly, Phys. Rev. A 29, 690 (1984).

[13] U. Gaubatz, P. Rudecki, S. Schiemann, and K. Bergmann, J. Chem. Phys. 92, 5363 (1990).

[14] M. Gottselig, D. Luckhaus, M. Quack, J. Stohner and M. Willeke, Helv. Chim. Acta 84, 1846 (2000).

[15] The $|1\rangle,|3\rangle,|4\rangle$ and $|5\rangle$ states correspond to torsional states with $0,2,3,5$ vibrational quanta, respectively. The $|2\rangle$ state is the first excited state of the asymmetric $S-D$ stretching mode.

[16] P. Král, Z. Amitay, and M. Shapiro, Phys. Rev. Lett. 89, 063002 (2002).

[17] T. Müller, K. B. Wiberg and P. H. Vaccaro, J. Phys. Chem. A 104, 5959 (2000). 